\title{
INVENTARIO PRELIMINAR DE AVES EN DOS FRAGMENTOS DE BOSOUE EN LA CORDILLERA ORIENTAL DE LOS ANDES COLOMBIANOS
}

\section{PRELIMINARY BIRD INVENTORY IN TWO FOREST FRAGMENTS OF THE COLOMBIAN EASTERN ANDEAN SLOPE}

Yudy Cristina Gomez ${ }^{1}$

Adelmo Rivera Díaz ${ }^{2}$ José Roberto Gómez ${ }^{3}$ Nelcy Patricia Vargas ${ }^{4}$

\section{RESUMEN}

La alteración en los bosques de Colombia aumenta constantemente en zonas donde no existen registros de su biodiversidad. Al realizar un inventario preliminar ornitológico en dos fragmentos de bosque al sur de la cordillera oriental de los andes colombianos sobre la vía Florencia-Suaza, en límites de los departamentos del Huila y del Caquetá, se evidencia el impacto ambiental negativo generado por el hombre. La avifauna fue registrada mediante observación directa y capturas con redes. Se registraron 229 individuos agrupados en 73 especies; las familias Thraupidae, Trochilidae y Tyrannidae fueron las más diversas. El análisis ecológico, a través del uso de hábitat y nicho, indicó que la mayoría de las especies se encontraron en el bosque primario y, en el tipo de dieta,

1 Trabajo de grado para obtener el título de Biólogo. Universidad de la Amazonia, Grupo de Investigación en fauna silvestre AKORE. Dirección para correspondencia: Calle 20 No $4^{\mathrm{a}}$ - 27 Florencia, Caquetá. E-mail: yunaves@gmail.com.

2 Trabajo de grado para obtener el título de Biólogo. Universidad de la Amazonía, Grupo de Investigación en Fauna Silvestre ANKORE, Dirección para correspondencia Cr 16 No 9-13, Florencia, Caquetá. E-mail: jemgmemo@gmail.com.

${ }^{3}$ Investigador independiente. Especialista en Gestión Ambiental, Biólogo, Universidad de la Amazonia, Doncello, Caquetá. E-mail: unmroberto@yahoo.es

${ }^{4}$ Investigador independiente. Especialista en Gestión Ambiental, Biólogo, Universidad de la Amazonia, Doncello, Caquetá. E-mail: patotus2004@yahoo.es los más abundantes fueron los insectívoros y frugívoros de sotobosque. Se amplió el rango de distribución altitudinal de cuatro especies: Heliodoxa schreibersii, Tangara xanthogastra, Xiphorhynchus erythropygius y Myiobius barbatus. El estado de conservación de los bosques es bueno, pero su constante fragmentación puede ocasionar la restricción y la migración local de la fauna, que depende de este ecosistema. La información presentada es el primer inventario de avifauna realizado para esta zona.

Palabras clave: Avifauna, especies, bosque, conservación.

\section{SUMMARY}

The alteration in the Colombian forests is constantly increasing in areas where no registers of its biodiversity exist. When carrying out a preliminary ornithological inventory in two forest fragments in the south of the eastern Colombian Andes Mountains on the Florencia-Suaza road, on the limit of the Departments of Huila and Caquetá, the negative environmental impact made by mankind was evident. The avifauna of this study was registered by direct observation and net captures. 229 individuals, grouped into 73 species were registered; the families Thraupidae, Trochilidae and Tyrannidae were the most diverse ones. The ecological analysis, through the use of habitat and niche, showed that the majority of species were found in the primary forest, and regarding the kind of diet, the most abundant ones were the insectivores and frugivores of sot forest. The altitudinal distribution range of four species was increased: Heliodoxa schreibersii, 
Tangara xanthogastra, Xiphorhynchus erythropygius and Myiobius barbatus. The state of conservation of forests is good, but its constant fragmentation can cause the restriction and local migration of the fauna that depends on this ecosystem. The information presented here is the first inventory of avifauna done for this zone.

Key words: Avifauna, species, forest, conservation.

\section{INTRODUCCIÓN}

Los bosques de la cordillera Oriental -vertiente sur este- presentan en la actualidad una de las mejores coberturas boscosas en los Andes; todavía es posible encontrar zonas donde existe continuidad de vegetación natural desde los páramos hasta las planicies de la Amazonía (Bohórquez, 2002). Sin embargo, algunos lugares han sido fragmentados, ocasionando una serie de consecuencias negativas que afecta a las especies silvestres.

La apertura del carreteable Florencia - Suaza y el establecimiento de familias colonizadoras, han transformado varias partes de este hábitat en pequeños parches de bosque relictuales y aunque los terrenos sobre los $1000 \mathrm{msnm}$ han sido declarados reserva forestal, los colonos efectúan prácticas extractivas de madera y quemas a los bosques para implantar cultivos agrícolas, lo que ha hecho evidente que se pueda crear un declinamiento en la diversidad biológica.

Durante las últimas décadas, el interés por la ecología de las comunidades de aves en el neotrópico ha sido notable (Serrano, 2000). Según May (1989), la avifauna, se constituye como uno de los mejores grupos para estudios biológicos en general y es considerada como un excelente bioindicador del estado de la conservación y la alteración de los ecosistemas (Figueroa, 1998; Ramírez E Ramírez, 2002). En las aves, su movilidad, fácil localización, observación y monitoreo permite realizar listados de especies que, analizados ecológicamente, revelan las condiciones ambientales de un área determinada, lo que permite establecer recomendaciones sobre la protección de su hábitat.

La destrucción y la alteración del medio ambiente, se empieza a reconocer en todo el mundo, especialmente en países como Colombia, donde la biología conservacionista apenas presenta las primeras alternativas reales de manejo
(Serrano, 2000). Estos acontecimientos son importantes en las regiones donde la riqueza de flora y fauna es tan grande, como es el caso de la región andina, considerada una de las zonas con mayor diversidad biológica, en especial, en áreas entre los 1000 y 2000msnm (Rangel, 1998), siendo, al mismo tiempo, una de las más amenazadas por la alteración de su hábitat (Kattan $\mathcal{E}$ Álvarez-López, 1996). En esta área, la fragmentación de los bosques se ha incrementado aceleradamente, aumentando su heterogeneidad y, por ende, la restricción o la desaparición de algunas especies, por el aislamiento poblacional en sus límites altitudinales y la irrupción en rutas de migración altitudinal (Kattan E Álvarez-López, 1994).

La perturbación del hábitat y la constante pérdida de la biodiversidad, aún desconocida, además de la escasa información existente en los archivos acerca de la zona, debido a los problemas de orden público que obstaculizan su estudio, crea la necesidad de realizar un inventario preliminar de la avifauna presente en dos fragmentos de bosque, sobre la vía Florencia - Suaza, con el objetivo de evaluar las avifauna presente en el bosque, permitiendo conocer el impacto ambiental ocasionado en esta área y así, formular estrategias de conservación para la fauna y flora que allí se alberga.

\section{MATERIALES Y MÉTODOS}

Área de estudio. El inventario, se realizó en dos fragmentos localizados en una franja altitudinal andina entre los 1350 y 1700 metros de elevación. Ambos fragmentos, se encuentran sobre la vía Florencia - Suaza, en límites de los departamentos del Huila y del Caquetá, al sur de la cordillera oriental de Colombia (Figura 1). Esta zona de vida corresponde a un bosque húmedo premontano y subtropical (Holdridge, 1967), el rango de temperatura oscila entre los 10 y $23^{\circ} \mathrm{C}$, la precipitación media anual es de $100-200 \mathrm{~cm} 3$ y existe una alta humedad relativa y nubosidad periódica.

Primer fragmento de monitoreo: Finca Marllins (1 $\left.49^{\prime} 11^{\prime \prime} \mathrm{N}-75^{\circ} 47^{\prime} 34^{\prime \prime} \mathrm{W}\right)$ : se encuentra ubicada en el kilómetro 57 sobre la vía Florencia - Suaza, en la vereda Alto Campo Hermoso, del municipio de Suaza, departamento del Huila, ocupando un área aproximada de 30ha. El bosque presenta una alta humedad, permanece nublado en las mañanas y, final de la tarde, con temperaturas que oscilan entre los 10 y $22^{\circ} \mathrm{C}$, existe 


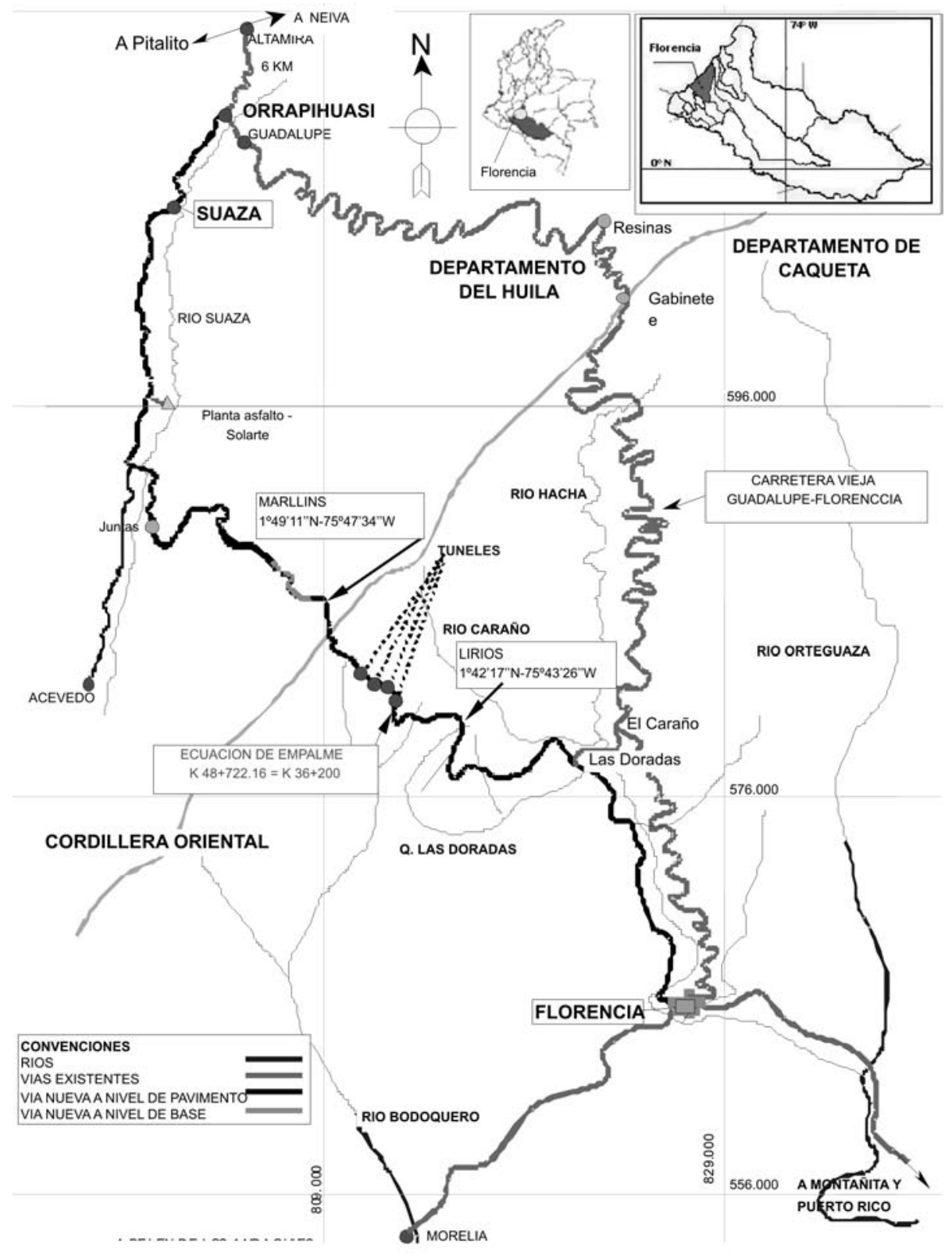

Figura 1. Localización del área de estudio (adaptado de INVIAS, 2000).

un buen estado de conservación en sus partes altas y, a medida que se acerca a la carretera, se observan más claros y transformación del bosque, por la extracción de madera y cultivos agrícolas.

Segundo fragmento de monitoreo: Finca los Lirios (142'17'N-7543'26”'W): ubicada en el kilómetro 28 sobre la vía Florencia - Suaza, en la vereda las Brisas, corregimiento el Caraño, del municipio de Florencia, departamento del Caquetá, en un área aproximada de 15ha, con temperaturas que van desde los 19 a $23^{\circ} \mathrm{C}$; el bosque presenta en su parte basal, una vegetación predominante de las familias Ericaceae y Melastomataceae (Marín-Vásquez \& Aguilar-Gonzáles, 2004), y se localiza en laderas con pendientes que incluyen algunos yacimientos de agua, pequeños relictos de bosques riparios y cultivos de café y plátano.

Se realizó una visita de cuatro días a cada fragmento a mediados y finales de abril del 2004. Con un esfuerzo 
de muestreo de 56 horas/hombre de cuatro personas y observaciones realizadas con binoculares $10 \mathrm{X}$ de aumento, se llevaron a cabo a través de recorridos por senderos ya trazados por los colonos, en los que se visitaron todos los hábitats de cada fragmento durante las mañanas, de 06:00 a 10:00 y, en las tardes, de las 15:00 a las 18:00 registrando todos los avistamientos sin un límite definido. Se realizaron capturas usando doce redes de niebla de 3 y $6 \mathrm{~m}$, las cuales, fueron extendidas en las mañanas: 06:30 a 10:30 y en las tardes: $15: 00$ a las 18:00, durante todas las visitas, en el interior del bosque (primario y secundario) y a lo largo de todos los senderos. Los individuos capturados fueron registrados, realizando anotaciones sobre su estado reproductivo y tomando algunas fotografías (cámara fotográfica CANON EOS 500n zoom 28 - 80), para obtener un registro completo; de igual modo un ejemplar por cada especie capturada fue depositado en el Museo de Historia Natural de la Universidad de la Amazonia UAM.

Los hábitat muestreados, se clasificaron de acuerdo a la metodología propuesta por Stiles E Bohórquez (2000), en: $\mathrm{BM}=$ sotobosque y dosel de bosque maduro; $\mathrm{BS}=$ bosque secundario intervenido, con algunos árboles de tipo primario que constituyen el dosel; BB = bordes de bosque con vegetación densa asociada a cuerpos de agua y áreas abiertas; $\mathrm{RB}=$ rastrojo bajo, vegetación arbustiva de crecimiento secundario. Se realizó una curva de acumulación de especies, siguiendo a Moreno (2001), en la cual, se registró la cantidad de especies acumulada a lo largo de las visitas, para evaluar la efectividad del inventario. Con base en las observaciones de algunas aves en actividad de forrajeo y según la literatura consultada (Hilty \& Brown 1986; Kattan et al. 1994; Kattan et al. 1996; Figueroa, 1998; Stiles \& Roselli, 1998; Stiles \& Bohorquez, 2000; Castaño, 2001, Hobson et al. 2003; Fierro- Calderón et al. 2006; Moreno-Salazar E Camargo-Martínez, 2008), se estableció la dieta de las aves, teniendo en cuenta los siguientes grupos alimenticios: CAR = Carnívoros; FRU $=$ Frugívoros; GRA = Granívoros; INS = Insectívoros; $\mathrm{NEC}=$ Nectarívoros y INS-FRU $=$ InsectívorosFrugívoros. Para la clasificación taxonómica de las aves, se empleó la guía de campo de Hilty $\&$ Brown (1986), el listado de Salaman et al. (2001), teniendo en cuenta los cambios en la nomenclatura de género y especie propuestos por Sibley \& Monroe (1990) y la AOU.

\section{RESULTADOS Y DISCUSIÓN}

Caracterización ecológica. Se registraron en total 229 individuos de 73 especies (Tabla 1), así: por observación directa, 147 individuos de 24 especies; en capturas con redes, 82 individuos de 37 especies y, por los dos métodos, se registraron doce especies. Se colectó un ejemplar de cada una de las 49 especies capturadas.

La curva de acumulación de especies muestra un incremento constante en el número de especies, a medida que se aumenta el esfuerzo de muestreo (Figura 2), no se aprecia una estabilización al final que caractericé una curva asintótica, lo que indica, que para esta comunidad, la curva se encuentra crecimiento.

La cantidad de especies en los diferentes tipos de hábitat de los sitios muestreados esta muy marcada: en los dos sitios, el $64 \%$ de las especies se encontraron en bosque maduro (BM), con especies en el interior del bosque y claros y un $14 \%$ de las especies en bosque secundario (BS), en especial en el sotobosque. En Marllins, para bordes de bosque (BB) y rastrojo de bajo (RB), solo el $11 \%$ de las especies se registró en cada categoría, mientras que en Lirios, el $21 \%$ de las especies, se encontró en rastrojo bajo (RB) y solo el $6 \%$ en bordes de bosque (BB).

Con un registro total de 73 especies, distribuidas en 61 géneros, 23 familias y nueve órdenes, siendo Paseriformes el orden que registró la mayor diversidad, con 14 familias, seguida de Piciformes con dos familias y, el resto de órdenes, con una familia. Las tres familias más diversas fueron: Tyrannidae (13 especies), con el 18\%; Thraupidae (12 especies), con el 16\% y Trochilidae y Furnariidae con 10 especies, con el $14 \%$ cada una; en el resto de las familias, su diversidad no fue mayor a cuatro especies. La familia Tyrannidae y Trochilidae fueron las más diversas en Marllins y la familia Traupidae en Lirios. La riqueza específica en Marllins fue de 47 especies y en Lirios de 32 especies, donde sólo seis especies están presentes en los dos sitios (Chordeiles acutipennis, Coeligena coeligena, Tangara parzudakii, Tangara xantocephala, Tyrannus melancholicus, Wilsonia canadensis).

Dieta alimenticia. La mayoría de las aves registradas (43\%), se alimentan de insectos, el $18 \%$ de cultivos de frutas, como mora (Rubus glaucus) y curuba (Passiflora mollisima) y frutas silvestres, de algunos géneros, como; Miconia, Panopsis, Peromia; otro $18 \%$ se alimenta de 
Tabla 1. Listado general de las especies de aves encontradas en las dos localidades.

\begin{tabular}{|c|c|c|c|c|c|}
\hline FAMILIA & ESPECIE & REGISTRO & HÁBITAT & $\begin{array}{c}\text { GRUPO } \\
\text { ALIMENTICIO }\end{array}$ & LOC \\
\hline \multirow{2}{*}{ ACCIPITRIDAE } & Buteo brachyurus & V & BS & CAR & $\mathrm{M}$ \\
\hline & B. albonotatus & V & BM & CAR & $\mathrm{L}$ \\
\hline CRACIDAE & Aburria aburri* & V & $\mathrm{BM}, \mathrm{BS}$ & FRU & $\mathrm{L}$ \\
\hline PSITTACIDAE & Pyirrhura melanura & C & BM & FRU & $M$ \\
\hline \multicolumn{6}{|l|}{ CUCULIDAE } \\
\hline Sub: Crotophaginae & Crotophaga ani & V & RB & INS & $\mathrm{L}$ \\
\hline STRIGIDAE & Megascops sp. & $\mathrm{V}$ & BM & CAR & $\mathrm{M}$ \\
\hline CAPRIMULGIDAE & Chordeiles acutipennis & C & BB & INS & $\mathrm{M}, \mathrm{L}$ \\
\hline \multicolumn{6}{|l|}{ TROCHILIDAE } \\
\hline Sub: Phaethornithinae & Phaethornis syrmatophorus & C & BM & NEC & M \\
\hline \multirow{9}{*}{ Sub: Trochilinae } & Doryfera ludoviciae & $\mathrm{C}$ & BM & NEC & $\bar{L}$ \\
\hline & Adelomyia melanogenys & $\mathrm{C}$ & BM & NEC & $\bar{M}$ \\
\hline & Eriocnemis alinae & $\mathrm{C}$ & $\mathrm{BM}$ & NEC & $M$ \\
\hline & Coeligena coeligena & $\mathrm{C}, \mathrm{V}$ & BM & NEC & $M, L$ \\
\hline & C. torquata & $\mathrm{C}$ & BM & NEC & $\mathrm{M}$ \\
\hline & Boissonneaua flavescens & $\mathrm{C}$ & BM & NEC & $M$ \\
\hline & Heliodoxa schreibersii ${ }^{*}$ & $\mathrm{C}$ & $\mathrm{BM}$ & NEC & $\mathrm{L}$ \\
\hline & H. rubinoides & $\mathrm{C}$ & $\mathrm{BM}$ & NEC & $\mathrm{M}$ \\
\hline & Campylopterus falcatus & $\mathrm{C}$ & $\mathrm{BB}$ & NEC & $\mathrm{M}$ \\
\hline RAMPHASTIDAE & Aulacorhynchus prasinus & $\mathrm{C}$ & $\overline{B M}$ & FRU & $M$ \\
\hline PICIDAE & Melanerpes formicivorus & $\mathrm{C}, \mathrm{V}$ & $\mathrm{BB}$ & INS & $\mathrm{M}$ \\
\hline \multicolumn{6}{|l|}{ FURNARIIDAE } \\
\hline \multirow{3}{*}{ Sub:Furnariinae } & Premnoplex brunnescens & $\mathrm{C}$ & $\overline{B M}$ & INS & $M$ \\
\hline & Syndactyla subalaris & $\mathrm{C}$ & BM & INS & $M$ \\
\hline & Thripadectes virgaticeps & $\mathrm{C}$ & $\mathrm{BM}$ & INS & $\mathrm{L}$ \\
\hline \multirow{7}{*}{ Sub: Dendrocopaltinae } & Dendrocincla tyrannina & $\mathrm{C}, \mathrm{V}$ & BM & INS & $\mathrm{L}$ \\
\hline & Glyphorynchus spirurus & $\mathrm{V}$ & BM & INS & $\mathrm{L}$ \\
\hline & $\begin{array}{c}\text { Xiphocolaptes } \\
\text { promeropirhynchus }\end{array}$ & $C, V$ & BM & INS & M \\
\hline & Dendrocolaptes picumnus & $\mathrm{C}$ & $\mathrm{BM}$ & INS & $\mathrm{M}$ \\
\hline & Xiphorhynchus erythropygius* & $\mathrm{C}$ & BS & INS & $M$ \\
\hline & $X$. triangularis & $\mathrm{C}$ & BS & INS & $\mathrm{M}$ \\
\hline & Lepidocolaptes souleyetii & $\mathrm{V}$ & BM & INS & $\mathrm{M}$ \\
\hline \multirow{2}{*}{ THAMNOPHILIDAE } & Drymophila caudata & $\mathrm{V}$ & $\mathrm{BM}$ & INS & $M$ \\
\hline & Pyriglena leuconota & $\mathrm{V}$ & $\mathrm{BM}$ & INS & $M$ \\
\hline \multirow{3}{*}{ PIPRIDAE } & Masius chrysopterus & $\mathrm{C}$ & $\mathrm{BB}$ & INS-FRU & $M$ \\
\hline & Lepidothrix coronata & $\mathrm{C}, \mathrm{V}$ & BS & INS-FRU & $\mathrm{L}$ \\
\hline & Xenopipo atronitens ${ }^{*}$ & $\mathrm{~V}$ & $\mathrm{BB}$ & INS-FRU & $\mathrm{L}$ \\
\hline \multirow{5}{*}{ TYRANNIDAE } & Zimmerius viridiflavus & $\mathrm{V}$ & BS & INS & $M$ \\
\hline & Mionectes striaticollis & $\bar{C}$ & $\mathrm{BB}$ & INS-FRU & $M$ \\
\hline & M. olivaceus & $\mathrm{C}$ & $\mathrm{BM}$ & INS-FRU & $\mathrm{L}$ \\
\hline & Leptopogon superciliaris & $\mathrm{V}$ & $\overline{B M}$ & INS-FRU & $\bar{L}$ \\
\hline & L. rufipectus & $\mathrm{V}$ & $\mathrm{BM}$ & INS-FRU & $M$ \\
\hline
\end{tabular}

Tabla 1. (continuación) 
Tabla 1. (continuación)

\begin{tabular}{|c|c|c|c|c|c|}
\hline & Myotriccus ornatus & $\mathrm{V}$ & BM & INS & $\mathrm{L}$ \\
\hline & Myobius sp. & C & BM & INS & $\mathrm{L}$ \\
\hline & M. barbatus* & C & BM & INS & $M$ \\
\hline & Myophobus flavicans & $\mathrm{C}$ & BM & INS & $\mathrm{M}$ \\
\hline & Sayornis nigricans & $\mathrm{C}$ & $\mathrm{BB}$ & INS & $M$ \\
\hline & Pyrrhomyias cinnamomeus & $\mathrm{C}, \mathrm{V}$ & $\mathrm{BB}$ & INS & $M$ \\
\hline & Myiotheretes fumigatus & $\mathrm{V}$ & BM & INS & $M$ \\
\hline & Tyrannus melancholicus & $\mathrm{V}$ & $\mathrm{RB}$ & INS & $\mathrm{M}, \mathrm{L}$ \\
\hline VIREONIDAE & Vireo leucophrys & $\mathrm{V}$ & BM & INS-FRU & $\mathrm{L}$ \\
\hline HIRUNDINIDAE & Stelgidopteryx ruficollis & $\mathrm{C}$ & $\mathrm{RB}$ & INS & $\mathrm{L}$ \\
\hline TROGLODYTIDAE & Henicorhina leucophrys & $\mathrm{C}$ & $\mathrm{BM}$ & INS & $M$ \\
\hline \multirow{4}{*}{ TURDIDAE } & Myadestes ralloides & $\mathrm{V}$ & BM & FRU & $\mathrm{M}$ \\
\hline & Catharus dryas & $\mathrm{V}$ & $\mathrm{BM}$ & FRU & $\mathrm{L}$ \\
\hline & C. ustulatus & $\mathrm{C}$ & BM & FRU & $\mathrm{L}$ \\
\hline & Turdus ignobilis & $\mathrm{V}$ & $\mathrm{RB}$ & INS & $\bar{L}$ \\
\hline \multirow{12}{*}{ THRAUPIDAE } & Tangara xanthogastra* & $\mathrm{C}$ & $\mathrm{BM}$ & FRU & $\mathrm{L}$ \\
\hline & T. nigroviridis & $\mathrm{C}$ & $\mathrm{BM}$ & FRU & $M$ \\
\hline & T. xantocephala & $\mathrm{C}, \mathrm{V}$ & $\mathrm{BM}$ & FRU & $\mathrm{M}, \mathrm{L}$ \\
\hline & T. parzudakii & $\mathrm{V}$ & $\mathrm{BM}$ & INS-FRU & $\mathrm{M}, \mathrm{L}$ \\
\hline & Ramphocelus carbo & $\mathrm{C}$ & $\mathrm{RB}$ & FRU & $\mathrm{L}$ \\
\hline & Thraupis episcopus & $\mathrm{C}$ & $\mathrm{RB}$ & INS-FRU & $\mathrm{L}$ \\
\hline & Anisognathus flavinucha & $\mathrm{C}, \mathrm{V}$ & BM & INS-FRU & $M$ \\
\hline & Diglossa albilatera & $\mathrm{C}$ & BS & NEC & $\mathrm{M}$ \\
\hline & D. cyanea & $\mathrm{V}$ & BS & FRU & $M$ \\
\hline & Coereba flaveola & $\mathrm{C}$ & BS & NEC & $\mathrm{L}$ \\
\hline & Chlorospingus ophthalmicus & $\mathrm{C}, \mathrm{V}$ & $\mathrm{BS}$ & INS-FRU & $M$ \\
\hline & Ch. flavigularis & $\mathrm{C}, \mathrm{V}$ & BM & INS-FRU & $\mathrm{L}$ \\
\hline EMBERIZIDAE & Zonotrichia capensis & $\mathrm{C}, \mathrm{V}$ & RB & GRA & $M$ \\
\hline \multirow{2}{*}{ CARDINALIDAE } & Cissopis leverianus & $\mathrm{C}$ & $\mathrm{RB}$ & INS & $\mathrm{L}$ \\
\hline & Piranga rubra* & $\mathrm{C}$ & BM & FRU & $\mathrm{M}$ \\
\hline \multirow{3}{*}{ PARULIDAE } & Wilsonia canadensis* & $\mathrm{V}$ & BM & INS & $\mathrm{M}, \mathrm{L}$ \\
\hline & Myioborus miniatus & $\mathrm{C}$ & BM & INS & $M$ \\
\hline & Basileuterus coronatus & $\mathrm{C}, \mathrm{V}$ & BM & INS & $M$ \\
\hline ICTERIDAE & Psarocolius decumanus & V & BS & FRU & $\mathrm{L}$ \\
\hline
\end{tabular}

Marllins (M) y Lirios (L). Abundancia C: común, PC: Poco común, E: escasa, O: ocasional. Registro: V: visual, C: captura. Hábitat: BM bosque maduro, BS: bosque secundario, BB: borde de bosque, RB: rastrojo bajo. Grupo alimenticio CAR: carnívoro, FRU: frugívoro, INS: insectívoro, NEC: nectarívoro, INS - FRUG: insectívoro - frugívoro, GRA: granívoro. LOC: Localidad; M: Marllins, L: Lirios. ( $\left.{ }^{*}\right)$ : Especies de interés

insectos y de frutas, seguida de los Nectarívoros (16\%), con doce especies, que incluyen dos aves pinchaflores (Coereba flaveola, Diglossa albilatera) y diez especies de colibríes, los demás grupos alimenticios presentan pocas especies y, en general, en los dos sitios, se puede ver que la distribución en cuanto a la dieta es muy similar (Tabla 2). En la relación de hábitat con los grupos alimenticios en Marllins y Lirios, las aves se encontraron, principalmente, en bosque maduro BM; las especies insectívoras e insectívoras-frugívoras ocurren a pequeñas zonas abiertas en el interior del bosque y sotobosque, las nectarívoras y frugívoras, estuvieron asociadas al dosel y sotobosque. En el bosque secundario (BS) y bordes de bosque (BB), las especies insectívoras se hallaron en el sotobosque y, las frugívoras, en el dosel. En rastrojo bajo (RB) donde se halla la única granívora, esta se encontró en un área de 


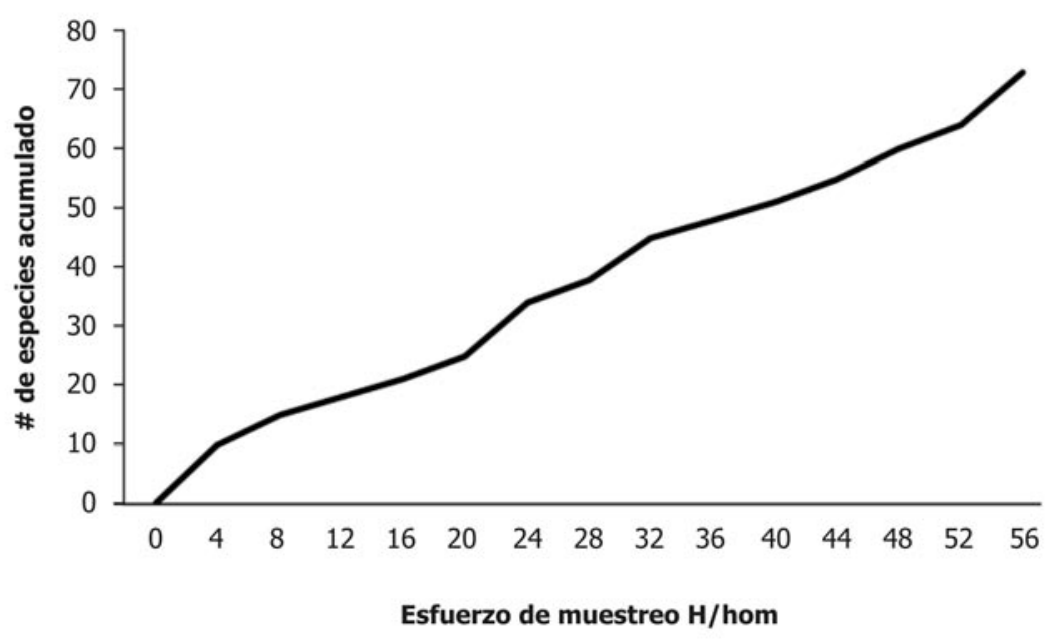

Figura 2. Curva de acumulación de especies durante el trabajo en campo.

pastizales y cultivos agrícolas. Las especies carnívoras, se observaron sobrevolando en los hábitats.

Especies de interés. 68 especies que representan el 93\% del total de las especies registradas en este estudio, presentaron un rango de distribución normal, según los reportes de Hilty E Brown (1986), Mazariegos (2000) y Salaman et al. (2001). Dos especies, Wilsonia canadensis y Piranga rubra, se albergan, de manera permanente, como migratorias boreales. Se amplía el rango de distribución altitudinal a 1350m en Lirios de: Heliodoxa schreibersii (colectado No. colección 006 UAM), restringida en Colombia al occidente de Putumayo y Vaupés hasta los $1000 \mathrm{~m}$, según Mazariegos (2000) y Tangara xanthogastra (colectado No. colección 0057 UAM), registrada hasta los $600 \mathrm{~m}$, en el occidente del Meta, Vaupés y la Amazonia, por Hilty \& Brown (1986) y Bohórquez (2002). En Marllins, se amplia el rango de distribución altitudinal a $1700 \mathrm{~m}$ de las especies: Xiphorhynchus erythropygius (colectado No. colección 0043 (IAM), registrado hasta $1500 \mathrm{~m}$, desde Panamá a Nariño a lo largo del occidente de la cordillera occidental y valle del Magdalena (Bohórquez, 2002) y Myiobius barbatus (colectado No. colección 0031 (IAM), reportada al occidente de la cordillera occidental, desde el golfo de Urabá hasta Nariño, al oriente de Colombia en la Macarena y sur oriente de Guainía, hasta los 1000m (Hilty E Brown, 1986; (Álvarez et al. 2003). Solo una especie (Aburra aburri), se encontró catalogada en bajo riesgo (Rengifo et al. 2002). Esta ave fue vista una vez en Lirios entre el sotobosque del bosque maduro y secundario.
La ejecución de inventarios rápidos constituye una herramienta útil en la evaluación del estado actual de la biodiversidad (Bohórquez, 2002), ya que permite evaluar el impacto ambiental que ocasiona, en este caso, la colonización y la construcción de vías, sobre las especies que son vulnerables a cambios drásticos en sus hábitat. Aunque este método esta sujeto a varias fuentes de error, principalmente, diferenciar las especies propias y visitantes, no tomar en cuenta la cantidad y el esfuerzo de muestreo y la variedad de hábitat presentes en un ecosistema (Remsen, 1994).

La composición taxonómica de la avifauna presenta algunos aspectos de interés, aunque incluye un gran número de especies de amplia distribución geográfica y ecológica (Marín Gómez, 2005). La ampliación del rango altitudinal de las cuatro especies (Heliodoxa schreibersii, Tangara xanthogastra, Xiphorhynchus erythropygius y Myiobius barbatus) permite establecer que este inventario, aunque es preliminar, arroja resultados interesantes, en cuanto a la distribución espacial de la avifauna en Colombia y confirma la necesidad e importancia de estudiar y conservar esta zona que, por la colonización, se esta deteriorando constantemente.

Inventarios ejecutados en la cordillera oriental de los Andes al sur y norte de Colombia, permiten realizar comparaciones en composición de especies para esta zona con los estudios realizados por Stiles $\mathcal{E}$ Roselli (1998), en un bosque alto andino, Stiles $\mathcal{E}$ Bohórquez 
Tabla 2. Número de especies por fragmento y porcentaje total, según su grupo alimenticio.

\begin{tabular}{|c|c|c|c|c|c|}
\hline Grupo alimenticio & Marllins & Lirios & Marllins y Lirios & Total & $\%$ \\
\hline Carnívoro & 2 & 1 & 0 & 3 & 4 \\
\hline Frugívoro & 7 & 7 & 1 & 13 & 18 \\
\hline Granívoro & 1 & 0 & 0 & 1 & 1 \\
\hline Insectívoro & 22 & 12 & 3 & 31 & 43 \\
\hline Nectarívoro & 9 & 4 & 1 & 12 & 16 \\
\hline Frugívoro- Insectívoro & 6 & 8 & 1 & 13 & 18 \\
\hline Total & 47 & 32 & 6 & 73 & 100 \\
\hline \multicolumn{6}{|c|}{ Se resta el número de especies encontradas en los dos sitios, según el grupo alimenticio. } \\
\hline Grupo alimenticio & Marllins & Lirios & Marllins y Lirios & Total & $\%$ \\
\hline Carnívoro & 2 & 1 & 0 & 3 & 4 \\
\hline Frugívoro & 7 & 7 & 1 & 13 & 18 \\
\hline Granívoro & 1 & 0 & 0 & 1 & 1 \\
\hline Insectívoro & 22 & 12 & 3 & 31 & 43 \\
\hline Nectarívoro & 9 & 4 & 1 & 12 & 16 \\
\hline Frugívoro- Insectívoro & 6 & 8 & 1 & 13 & 18 \\
\hline Total & 47 & 32 & 6 & 73 & 100 \\
\hline \multicolumn{6}{|c|}{ Se resta el número de especies encontradas en los dos sitios, según el grupo alimenticio. } \\
\hline Grupo alimenticio & Marllins & Lirios & Marllins y Lirios & Total & $\%$ \\
\hline Carnívoro & 2 & 1 & 0 & 3 & 4 \\
\hline Frugívoro & 7 & 7 & 1 & 13 & 18 \\
\hline Granívoro & 1 & 0 & 0 & 1 & 1 \\
\hline Insectívoro & 22 & 12 & 3 & 31 & 43 \\
\hline Nectarívoro & 9 & 4 & 1 & 12 & 16 \\
\hline Frugívoro- Insectívoro & 6 & 8 & 1 & 13 & 18 \\
\hline Total & 47 & 32 & 6 & 73 & 100 \\
\hline
\end{tabular}

(2000), en la serranía de los Quinchas en Boyacá, Bohórquez (2002), en la vertiente oriental de los Andes de Colombia y por Salaman et al. (1999), en la serranía de los Churumbelos en el Cauca. Las comparaciones, se realizaron con la información de registros entre los 1350 y 1700m. La mayoría de las especies son comparables con los otros sitios, a pesar de las diferencias en la distribución geográfica, la topografía de las vertientes de la cordillera y el grado de heterogeneidad de los tipos de bosque. La avifauna corresponde a la que se reporta en esta zona normalmente, lo que indica que ese estudio arrojó resultados representativos y que la riqueza de especies es bastante alta respecto al poco esfuerzo de muestreo realizado en comparación con las intensidades de muestreo en los otros trabajos.

En los dos sitios de muestreo, la mayor diversidad de aves, se halló en el bosque maduro (BM), con una gran 
cantidad de especies que dependen del sotobosque y áreas abiertas. Este bosque presenta una mayor cobertura en la zona (77\%) y bajo grado de intervención; su complejidad estructural ofrece una multitud de microhábitat que ofrecen diferentes actividades de forrajeo para las aves y otros grupos de animales (algunos anfibios y murciélagos), que son exclusivos de este ambiente como residentes permanentes o migratorios.

La curva de acumulación de especies, se constituye como un indicador de la efectividad y lo completo de un inventario. En este estudio, la curva de acumulación, se muestra en todo momento ascendente y lejos de alcanzar un comportamiento asintótico. El número de especies encontradas a lo largo del muestreo indica que aún faltan muchas especies por ingresar y que el inventario no esta completo, por lo cual, es prioritario continuar y completar el estudio para establecer criterios más específicos sobre el estado de la avifauna; sin embargo, es conveniente tener en cuenta la llegada de aves migratorias y los periodos de explosión de recursos vegetales, como épocas de florecimiento y fructificación.

El alimento es uno de los componentes primarios del nicho y, consecuentemente, un elemento importante (Ramírez E Ramírez, 2002), para determinar las interrelaciones ecológicas y cambios estaciónales de las aves. Un alto número de especies insectívoras y frugívoras muestra la abundancia de recursos alimenticios, en los diferentes hábitat. En la zona de estudio, los insectívoros predominan sobre los demás. La destrucción de la matriz vegetal crea zonas abiertas que promueven la proliferación y la abundancia de los insectos en los bordes de bosque y claros (Quevedo, 2002), ocasionando que los límites de estas áreas abiertas incrementen la oferta de alimento para los insectos, como lo es la materia orgánica restante de la extracción de madera y, en algunos casos, el establecimiento de pequeños cultivos agrícolas, lo que aumenta la disponibilidad alimenticia para las aves insectívoras. De igual modo, la abundancia de frutos en el dosel y sotobosque de los diferentes hábitats y los cultivos de frutas establecidos por los colonos cerca del bosque, muestra la disponibilidad de alimento para las aves frugívoras y la movilidad de estas especies entre los hábitats. Sin embargo, para muchas aves sensibles a los cambios en su hábitat, la oferta alimenticia disminuye.

En la avifauna de climas templados y zonas montañosas los movimientos altitudinales están asociados a la variación del clima y disponibilidad de alimento de sistemas montañosos (Hobson et al. 2003), estos movimientos son especialmente frecuentes en las aves nectarívoras (Stiles, 1988). La floración en la zona esta marcada estacionalmente, con un pequeño porcentaje observado en los bosques, la oferta alimenticia de los colibríes disminuye, ocasionando la movilidad altitudinal de estas especies de manera periódica o permanente, pues a excepción de las dos especies pincaflores, el número de nectarívoros en los dos sitios es relativamente bajo, teniendo en cuenta la diversidad que existe en esta franja altitudinal.

La importancia de la conservación de esta zona condiciona a que las comunidades de aves neotropicales que se caracterizan por su alta riqueza y estructura de especies raras con densidades poblacionales muy bajas (Karr et al. 1990; Leve E Stiles, 1994; Thiollay, 1994, citados por Kattan et al. 1996) permanezcan como elemento funcional del ecosistema, pues cambios estructurales en estos bosques causarían alteraciones en los diferentes hábitat, como la extinción local de la avifauna y otras especies vulnerables a la presión antrópica.

\section{CONCLUSIONES}

Los cambios de la distribución actual de las especies por hábitat, tanto en sus poblaciones como en la comunidad en general, así como a las áreas cercanas al carreteable, indican el impacto negativo que se ejerce sobre el ecosistema. Las actividades antrópicas, como la tala constante de árboles, quema del sustrato boscoso, el incremento de los cultivos agrícolas (café, mora, granadilla) y colonización en la zona, ocasiona lentamente la pérdida de muchos recursos sensibles a los cambios o alteraciones en sus hábitat, los cuales, son vitales para supervivencia y conservación del equilibrio del ecosistema.

Las áreas de la vertiente sur oriental de la cordillera de los Andes son consideradas como las mejor conservadas y es importante que se empleen actividades de conservación y de protección en esta zona, en especial en los parches de bosque y áreas donde aún persiste matriz vegetal intacta, ya que las especies registradas, se albergan en estos sitios y aunque este lugar es considerado como una zona de conservación por el Ministerio del Medio Ambiente, las constantes perturbaciones antrópicas aumentan la fragmentación de los bosques, disminuyendo su biodiversidad. 


\section{RECOMENDACIONES}

Este inventario careció de más esfuerzo de muestreo; sin embargo, los resultados presentados por ser uno de los primeros para esta zona sugieren que sea crítica para la conservación y que se realicen inventarios posteriores que promuevan la preservación de la flora y la fauna.

Es necesario aprovechar, de manera inmediata, toda oportunidad de crear y de fortalecer bases de datos sobre estos aspectos e iniciar proyectos de monitoreo a largo plazo, que permitan conocer constantemente el estado de conservación de esta zona.

\section{BIBLIOGRAFÍA}

ÁLVAREZ, M.; UMAÑA, A.M; MEJIA, G.D; CAJIAO, J; HILDEBRAND, V.P; GAST, F. 2003. Aves del Parque Natural Serranía del Chiribiquete, Amazonia- Provincia de la Guyana, Colombia. Biota Colombiana. 4(1): 49-63.

BOHÓRQUEZ, C.I. 2002. La avifauna de la vertiente oriental de los Andes de Colombia. Tres evaluaciones en elevación subtropical. Rev. Acad. Col. de Ciencias Físicas, Exactas y Naturales. 26(100):419-442.

CASTAÑO, G.J. 2001. Inventario preliminar de aves y mamíferos presentes en fragmentos boscosos en el San Jorge medio, departamento de Córdoba Colombia. Revista Ingeniería Forestal de la Universidad Nacional sede Medellín. Disponible desde Internet en: http://www.colforest.com/revista/vol13/articulocastano.html. (con acceso 08/08/01).

FIERRO-CALDERÓN, K; ESTELA, F.A; CHACONULLOA, P. 2006. Observaciones sobre las dietas de algunas aves de la cordillera oriental de Colombia a partir del análisis de contenidos estomacales. Ornitología Colombiana. 4:6-15.

FIGUEROA, A.A. 1998. Caracterización de la avifauna en cinco localidades del proyecto vial carretera alterna Buga-Buenaventura: Tramo MadroñalCórdoba-Valle del Cauca. Cespedesia (Colombia). 23(71-72):85-115.

HILTY, S.L.; BROWN, W.L. 1986. A guide to the birds of Colombia. Princeton University Press. (USA.). 836p.
HOBSON, K.A.; WASSENAAR, L.I.; MILA, B.; LOVETTE, I.; DINGLE, C.; SMITH, T.B. 2003. Stable isotopes as indicators of altitudinal distributions and movements in an Ecuadorean hummingbird community. Oecologia (USA). 136: 302-308.

HOLDRIDGE, L.R.1967. Life Zone Ecology. Tropical Science Center. San José (Costa Rica). 206p.

KATTAN, H.G.; SERRANO, V.H.; APARICIO, A. 1996. Aves de Escarlete: diversidad, estructura trófica y organización social. Cespedesia. 21(68) 9-17.

KATTAN, H.G.; ÁLVAREZ-LÓPEZ, H. 1994. Forest Fragmentation and Birds Extinctions: San Antonio Eighty Years Later. Conservation Biology (Colombia). 8(1):138-146.

KATTAN, H.G.; ÁLVAREZ-LÓPEZ, H. 1996. Preservation and management of biodiversity in fragmented landscapes in the Colombian Andes. En: Sichelhas, J.; Greenberg, R. (eds.) Forest parches in tropical landscapes (Wachington DC ). p.3-18.

MARÍN GÓMEZ, O.H. 2005. Avifauna del Campus de la Universidad del Quindio. Boletín SAO. 15:19p.

MARÍN-VÁSQUEZ, A.; AGUILAR-GONZÁLES, A.V. 2004. Diversidad de murciélagos en un gradiente altitudinal en la vertiente oriental de la cordillera oriental de los andes colombianos. Tesis de Biología. Universidad de la Amazonía. Florencia- Caquetá. 92p.

MAY, R.M. 1989. ¿How many species are there on Earth? Science. 241(1):1441-1449.

MAZARIEGOS, L.A. 2000. Colibríes de Colombia. En: Mazariegos, L.A. ed. Joyas Aladas de Colombia. Eds. Sociedad Antioqueña de Ornitología (Medellín). p.214-223.

MORENO, C.E. 2001. Métodos para medir la Biodiversidad. Manuales y Tesis SEA. (España)1:86p

MORENO-SALAZAR, N.; CAMARGO-MARTÍNEZ, P. 2008. Inventario preliminar de la avifauna de la vereda "Acuapal" municipio de Sasaima - Cundinamarca. Boletín SAO.18:15p. 
QUEVEDO, G.A. 2002. Composición taxonómica y algunos acontecimientos ecológicos sobre la avifauna observada en el municipio de Falán, departamento del Tolima. Boletín informativo mensual de Proaves (Bogotá). Disponible desde Internet en: http://www.proaves.org/aleteo4.html. (con acceso 12/11/04).

RAMÍREZ, J.E.; RAMÍREZ, M.G. 2002. Avifauna del la región del oriente de la sierra de Huautia: Morelos, México. Revista de la Universidad Autónoma de México. Serie Zoología. 78(1):91-111.

RANGEL-CH, J.O. 1998. Diversidad de Biota. En: Chávez, M.E.; Arango, N. (eds). Informe nacional sobre el estado de la biodiversidad. Eds. Instituto de Investigaciones Alexander von Humboldt. PNUMA. Ministerio de Medio Ambiente (Santa Fe de Bogotá). 3:316-318.

REMSEN, J.V. 1994. Use and misuse of bird lists in community ecology and conservation. Auk (USA). 111:225-227.

RENJIFO, L.M.; FRANCO-MAYA, A.M.; AMAYA-ESPINEL, J.D.; KATTAN, G.H.; LÓPEZ-LANÙS, B. (eds.). 2002. Libro rojo de aves de Colombia. Serie Libros Rojos de Especies Amenazadas de Colombia. Eds. Instituto de Investigación de Recursos Biológicos Alexander von Humboldt y Ministerio del Medio Ambiente. Bogotá, Colombia. 554p.

SALAMAN, P.G.W.; DONEGAN, T.M.; CUERVO, A.M. 1999. Ornithological surveys in Serranía de los Churumbelos, south Colombia. Cotinga. 12:29-39.
SALAMAN, P.G.W.; CUADROS, T.; JARAMILLO, J.G.; WEBER, W. (eds). 2001. Lista de chequeo de las aves de Colombia. Edit.. Soc. Antioqueña de Ornitología (Medellín). 116p.

SERRANO, V.H. 2000. Informe de las aves presentes en la finca El Roble. New Birds (Bogotá). Disponible desde Internet en: http://www.cafemesadelossantos.com/organic/2000_serrano.html. (con acceso 12/11/04).

SIBLEY, C.G.; MONROE, B.L. Jr. 1990. Distribution and Taxonomy of Birds of the World. Yale University Press (New Haven and London). 1111p.

STILES, F.G. 1988. Altitudinal movements of birds on the Caribbean slope of Costa Rica. En: Almeda, F.; Pringle, C. (eds). Tropical rainforests: diversity and conservation. Edit. California Academy of Sciences (San Francisco). p.243-258.

STILES, F.G.; ROSSELLI, L. 1998. Inventario de las aves del bosque altoandino: comparación de dos métodos. Caldasia. 20:29-43.

STILES, F.G.; BOHÓRQUEZ, C.I. 2000. Evaluando el estado de la biodiversidad: El caso de la avifauna de la serranía de los Quinchas Boyacá, Colombia. Caldasia (Colombia). 22(1):61-92.

Recibido: Mayo 15 de 2008

Aceptado: Septiembre 9 de 2008 